\title{
Investigation of SARS-CoV-2 in Semen of Patients in the Acute Stage of COVID-19 Infection
}

\author{
Bircan Kayaaslan $^{a}$ Gulay Korukluoglu ${ }^{b}$ Imran Hasanoglua Ayse Kaya Kalem $^{a}$ \\ Fatma Eser $^{\mathrm{a}}$ Esragul Akinci $^{\mathrm{c}}$ Rahmet Guner ${ }^{\mathrm{a}}$ \\ a Infectious Disease and Clinical Microbiology, Ankara Yildirim Beyazit University, Ankara City Hospital,

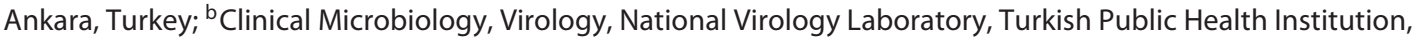 \\ Ankara, Turkey; ' Infectious Disease and Clinical Microbiology, University of Health Sciences, Ankara City \\ Hospital, Ankara, Turkey
}

\section{Keywords}

SARS-CoV-2 · COVID-19 · Semen · PCR · Sexual transmission

\begin{abstract}
Introduction: The presence of new coronavirus (SARS(oV-2) in semen and the possibility of sexual transmission have become new subjects of curiosity. There is a discrepancy regarding this issue in the literature. The presence of SARS-CoV-2 in semen has been investigated in a limited number of studies, and mostly in recovering patients. We aimed to investigate the presence of SARS-CoV-2 RNA in semen of patients with a positive nasopharyngeal swab test for SARS-CoV-2 in the acute stage. Methods: We enrolled adult male patients who were hospitalized with confirmed SARSCOV-2 infection in the study. In addition to routine laboratory and radiological tests, semen sample was obtained from volunteers and transferred to the Turkish Public Health Institution, National Virology Laboratory. The samples were processed for the detection of SARS-CoV-2 RNA on the day of collection. Results: Sixteen patients were included in the study. The median age was 33.5 years (18-54). All but one had respiratory symptoms. None of the patients had a history or symptoms of urogenital disease. All semen samples
\end{abstract}

were obtained during hospitalization and in the acute stage of the infection. The median time to obtain a semen sample after positive nasopharyngeal test was 1 day (0-7). All semen samples were detected as negative for SARS-CoV-2 PCR. Discussion/Conclusion: Although all semen samples were obtained in acute stage of the infection when the nasopharyngeal swab test was positive, we did not detect SARS-CoV-2 in semen. The results of our study support the thought that sexual transmission via semen does not have an important role in the person-to-person transmission of SARS-CoV-2. We think that our study will provide new information to fill the gap in the literature.

(c) 2020 S. Karger AG, Basel

\section{Introduction}

Severe acute respiratory syndrome coronavirus-2 (SARS-CoV-2) is a new pandemic virus, and many aspects are still unknown or little researched. Presence/absence of new coronavirus in sperm and its effect on the male genital system is a new subject of curiosity. This issue is also critical in terms of determination of the possibility of transmission by sexual contact and the infec-

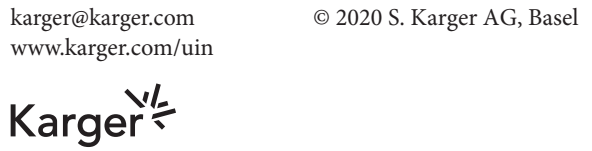

Bircan Kayaaslan

Infectious Disease and Clinical Microbiology

Ankara Yildirim Beyazit University, Ankara City Hospital

Bilkent Street no:1, TR-06800 Ankara (Turkey)

drbican@gmail.com 
tion risk during genitourinary implementations. New lines of evidence suggest that non-respiratory system involvement and other transmission routes may be possible during infection $[1,2]$. Demonstration of angiotensinconverting enzyme 2 (ACE2) receptor expression, one of the major receptors of SARS-CoV-2, in testicular tissue increased curiosity about testicular involvement and possible transmission by sexual contact [3]. Although SARS$\mathrm{CoV}-2$ has been investigated in different types of clinical specimens, the studies regarding presence of SARS$\mathrm{CoV}-2$ in semen are limited, and mostly in recovering patients [4-6]. Thus, we aimed to investigate the presence of SARS-CoV-2 RNA in semen in patients with positive nasopharyngeal swab test for SARS-CoV-2 in the acute stage of the infection.

\section{Materials and Methods}

This study was conducted between the 20 and 25 of April 2020 in Ankara City Hospital. Laboratory-confirmed coronavirus disease (COVID-19) patients older than 18 years who were hospitalized in COVID-19 wards (isolation units) were included in the study. Severe patients were excluded. Demographic and clinical characteristics of the patients were collected prospectively. Laboratory tests (complete blood count, biochemical tests, C-reactive protein, procalcitonin), computed tomography and nasopharyngeal swab test for SARS-CoV-2 RNA were routinely performed. The patients who had a positive nasopharyngeal swab test for SARS-CoV-2 taken on admission underwent consecutive polymerase chain reaction (PCR) tests. Semen samples were collected from volunteers. A written informed consent was taken from the patients before semen collection. The patients were informed about the appropriate semen collection procedure (washing hands and penis with soap and water, then drying of hands and penis with paper towels, and then avoiding touching any surface). Semen samples were collected into sterile containers and transferred to Turkish Public Health Institution, National Virology Laboratory with a sealed sterile transport system under cold chain rules and processed for detection of SARS-CoV-2 RNA.

Samples were processed on the day of collection. After centrifugation of urine samples at 3,000 rpm for $5 \mathrm{~min}$, the supernatant was removed from the medium, and $1 \mathrm{~mL}$ viral transport medium (VTM; MEM supplemented with penicillin-streptomycin and bovine serum albumin [BSA]) was added to the pellet, and vortexed, homogenized, and transferred to $2 \mathrm{~mL}$ volumes of cryovial tubes. Sperm samples were homogenized by vortexing after the addition of $1 \mathrm{~mL}$ of VTM and transferred to cryovial tubes. The samples, whose registration was completed, were immediately taken for extraction.

Extraction was performed with the Nucleic Acid Isolation Kit from R to M14 clinical sample (34 RN-N-14-111-100; R \& D Ltd., Turkey) and RINA-M14 Nucleic Acid Isolation Robot. The PCR process was performed with the Bio-Speedy ${ }^{\circledR}$ COVID-19 RTqPCR Detection Kit (Cat. No. BS-SY-WC-305, Bioeksen; R\&D Ltd., Turkey), and samples were enrolled in a real-time PCR instrument, Bio-Rad CFX96 Touch ${ }^{\mathrm{TM}}$ (Bio-Rad Laboratories Inc.,
USA). In the evaluation of the results, the proliferation curves obtained in the FAM/HEX channels were examined. Non-sigmoidal curves were recorded as negative. In cases in which positive, negative, and internal control values met appropriate criteria, if $\mathrm{Ct}<40$, the result was considered positive.

\section{Statistical Analyses}

All statistical analyses were performed using SPSS statistical software version 18.0 (IBM Corp., Armonk, NY, USA). Number and their percentages were used for categorical variables. Median and range (minimum-maximum or interquartile range [IQR]) were used for numerical variables.

\section{Results}

Of 149 patients admitted to the COVID-19 wards (isolation units) with pre-diagnosis of COVID-19 infection during the study period, 56 (37.5\%) had a positive nasopharyngeal SARS-CoV-2 PCR. Twenty-eight male patients were asked whether they would like to volunteer to provide samples for the detection of SARS-CoV-2 in semen; 16 of them agreed to participate and were included in the study. The median age was $33.5(18-54)$ years. Of the patients, $11(68.7 \%)$ had a mild disease, others (31.3\%) had moderate disease (pneumonia) at the time of disease confirmation. None of the patients had a recent history and/or symptoms of urogenital disease. Leukopenia, lymphopenia, and thrombocytopenia were detected in 1, 2, and 1 case, respectively. Ground-glass opacities were the most frequently detected radiological changes (68.7\%). None of them developed complications. Only one patient experienced clinical deterioration and was transferred to the intensive care unit during the follow-up. All patients survived. Baseline characteristics of the 16 patients enrolled the study are shown in Table 1.

All seminal fluid was collected during the hospitalization and in the acute stage of the disease. Median time from a positive nasopharyngeal swab test to obtaining semen sample was 1 day (0-7). Of 16 semen samples, 4 were provided on the same day as positive nasopharyngeal swab test, and 6 were provided on the day before and after the positive nasopharyngeal swab. Only 2 samples were provided on day 7 after positive nasopharyngeal swab. The results and the time from positive nasopharyngeal test to semen collection are shown in Table 2. The timeline of consecutive nasopharyngeal swab and semen sample test for SARS-CoV-2 PCR is shown in Figure 1. All semen sample tests were detected as negative for SARSCoV-2. In 5 patients, SARS-CoV-2 positivity of the nasopharyngeal swab test persisted even after a negative test of seminal fluid. 
Table 1. Baseline characteristics of patients infected with SARSCOV-2

\begin{tabular}{|c|c|}
\hline Age, median (min-max), years & $33.5(18-54)$ \\
\hline Underlying disease $^{\mathrm{a}}$ & $2(12.5)$ \\
\hline \multicolumn{2}{|l|}{ Epidemiological history } \\
\hline (exposure to SARS-CoV-2-positive person) & $11(68.8)$ \\
\hline \multicolumn{2}{|l|}{ Symptoms } \\
\hline Fever & $6(35.5)$ \\
\hline Cough & $7(43.7)$ \\
\hline Dyspnea & $1(6.3)$ \\
\hline Sore throat & $1(6.3)$ \\
\hline Fatigue & $9(56.3)$ \\
\hline Headache & $3(18.8)$ \\
\hline Myalgia/arthralgia & $5(31.2)$ \\
\hline \multicolumn{2}{|l|}{ Laboratory findings } \\
\hline Leukopenia $\left(<4 \times 10^{9} / \mathrm{L}\right)$ & $1(6.3)$ \\
\hline Lymphocyte $\left(<1.1 \times 10^{9} / \mathrm{L}\right)$ & $2(12.5)$ \\
\hline Neutrophil/lymphocyte ratio & $0.7 / 4.2$ \\
\hline Thrombocytopenia $\left(<150 \times 10^{9} / \mathrm{L}\right)$ & $1(6.3)$ \\
\hline Elevated aspartate transaminase $(>35 \mathrm{U} / \mathrm{L})$ & $3(18.8)$ \\
\hline Elevated alanine transaminase $(>45 \mathrm{U} / \mathrm{L})$ & $5(31.2)$ \\
\hline Increased creatinine $(1.3 \mathrm{mg} / \mathrm{dL})$ & - \\
\hline Increased lactate dehydrogenase (>246 U/L) & $2(12.5)$ \\
\hline Increased creatine kinase (>294 U/L) & $2(12.5)$ \\
\hline Increased C-reactive protein $(>0.005 \mathrm{~g} / \mathrm{L})$ & $8(50)$ \\
\hline \multicolumn{2}{|l|}{ Chest computed tomography findings } \\
\hline Ground-glass opacities & $11(68.8)$ \\
\hline Consolidation & $2(12.5)$ \\
\hline \multicolumn{2}{|l|}{ Disease severity } \\
\hline Mild disease & $5(31.2)$ \\
\hline Pneumonia & $11(68.8)$ \\
\hline Urogenital infection history & - \\
\hline Urogenital symptoms & - \\
\hline Positivity of SARS-CoV-2 PCR in semen & - \\
\hline Interval between positive nasopharyngeal & \\
\hline swab and semen test, median (IQR), days & $1(0-4)$ \\
\hline Presence of sexually transmitted disease & - \\
\hline Outcome (survived) & $16(100)$ \\
\hline
\end{tabular}

Data are presented as $n(\%)$ unless noted otherwise. ${ }^{a}$ Obesity and renal transplantation.

\section{Discussion}

SARS-CoV-2, the new coronavirus, has infected more than 13 million people, and we are still learning new information about it [7]. Although the main concern is severe infections that result in death, the fact that a large number of people are infected has revealed the need to address different aspects of the infection. Infection of systems other than the respiratory site and other routes of transmission are the main ones. SARS-CoV-2 primarily affects the respiratory system and is mainly transmitted by respiratory droplets and the close contact to secretions
Table 2. Time from a positive nasopharyngeal swab test to semen sample collection, and SARS-CoV-2 PCR results in laboratoryconfirmed COVID-19 patients

\begin{tabular}{|c|c|c|c|}
\hline Patient & $\begin{array}{l}\text { Age, } \\
\text { years }\end{array}$ & $\begin{array}{l}\text { Time from swab test to } \\
\text { semen collection, days }\end{array}$ & $\begin{array}{l}\text { Result of SARS- } \\
\text { CoV-2 PCR }\end{array}$ \\
\hline 1 & 45 & Same day & Negative \\
\hline 2 & 18 & 1 & Negative \\
\hline 3 & 24 & 1 & Negative \\
\hline 4 & 36 & 7 & Negative \\
\hline 5 & 53 & 4 & Negative \\
\hline 6 & 54 & 1 & Negative \\
\hline 7 & 41 & 4 & Negative \\
\hline 8 & 46 & 4 & Negative \\
\hline 9 & 27 & Same day & Negative \\
\hline 10 & 35 & 4 & Negative \\
\hline 11 & 31 & 1 & Negative \\
\hline 12 & 22 & 1 & Negative \\
\hline 13 & 32 & Same day & Negative \\
\hline 14 & 44 & 7 & Negative \\
\hline 15 & 18 & 1 & Negative \\
\hline 16 & 25 & Same day & Negative \\
\hline
\end{tabular}

of the patients with COVID-19 [8]. New evidence indicates that involvement of other systems can appear during the SARS-CoV-2 infection [9-11]. It has been shown that SARS-CoV-2 RNA was present in different sample types other than respiratory samples such as urine, blood, and anal swabs $[12,13]$. The demonstration of the SARSCoV-2 viremia created concern about the possibility of the virus crossing the blood testicle barrier and invading the male genital system $[7,8,14-16]$. Additionally, the description of ACE2 receptors in human testis Leydig and seminiferous tubule cells increased the concern in this regard [3].

Sexual transmission of SARS-CoV-2 has been under investigation from the early time of the pandemic, and the presence/absence of SARS-CoV-2 in semen has been studied in case series. However, the findings of these studies have revealed conflicting results $[4,5,6,17-19]$. In fact, most of the studies investigating SARS-CoV-2 in semen reported that the virus could not be detected in semen [5, 17-19]. First, it was reported that SARS-COV-2 was not detected in semen of a 31-year-old man in the recovery period [4]. Later, small case series were reported to support this finding [5, 17-19]. Pan et al. [5] reported that they did not detect SARS-CoV-2 in the semen of 34 Chinese male patients recovering from COVID-19. However, collecting semen samples after patients recover is a major limitation of the study. The median time from a confirmatory diagnosis of COVID-19 to collection of se- 


\begin{tabular}{|c|c|c|c|c|c|c|c|c|c|c|c|c|c|}
\hline \multirow[b]{2}{*}{ Patients } & \multicolumn{13}{|c|}{ Days from admission to hospital } \\
\hline & Day 1 & Day 2 & Day 3 & Day 4 & Day 5 & Day 6 & Day 7 & Day 8 & Day 9 & Day 10 & Day 11 & Day 12 & Day 13 \\
\hline 1 & $\Delta \square$ & & $\triangle$ & & $\triangle$ & & & & & & & & \\
\hline 2 & $\Delta$ & & & & $\Delta$ & $\square$ & $\triangle$ & & $\triangle$ & & & & \\
\hline 3 & $\Delta$ & & & & $\triangle$ & $\square$ & $\Delta$ & & & $\triangle$ & & $\triangle$ & \\
\hline 4 & $\triangle$ & & & & & $\Delta$ & & & & & & & $\square$ \\
\hline 5 & $\Delta$ & & & & $\square$ & & & $\triangle$ & & & & $\triangle$ & \\
\hline 6 & $\Delta$ & $\square$ & $\Delta$ & & & $\triangle$ & $\triangle$ & & & & & & \\
\hline 7 & $\triangle$ & & & & & $\Delta$ & & & $\Delta$ & $\triangle$ & $\triangle$ & & $\square$ \\
\hline 8 & $\Delta$ & & & & & $\Delta$ & & $\Delta$ & & $\triangle$ & $\triangle$ & $\square$ & \\
\hline 9 & $\Delta \square$ & $\triangle$ & & $\triangle$ & & & & & & & & & \\
\hline 10 & $\Delta$ & & & & $\Delta$ & . & & & $\Delta$ & & $\triangle$ & $\triangle$ & $\square$ \\
\hline 11 & $\triangle$ & $\square$ & & & $\triangle$ & $\triangle$ & & $\triangle$ & $\triangle$ & & & & \\
\hline 12 & $\Delta$ & $\square$ & & & $\triangle$ & $\Delta$ & & $\triangle$ & $\triangle$ & & & & \\
\hline 13 & $\Delta$ & & & & $\Delta \square$ & & & & & & & & \\
\hline 14 & $\Delta$ & & & & & $\triangle$ & & $\triangle \square$ & & & & & \\
\hline 15 & $\Delta$ & & & & & $\triangle$ & & $\Delta$ & $\square$ & $\Delta$ & & & \\
\hline 16 & $\Delta \square$ & & & & $\triangle$ & & $\triangle$ & & & & & & \\
\hline \multicolumn{14}{|c|}{ A: $\quad$ Positive nasopharyngeal test for SARS-CoV-2 } \\
\hline \multicolumn{14}{|c|}{$\triangle: \quad$ Negative nasopharyngeal test for SARS-CoV-2 } \\
\hline \multicolumn{14}{|c|}{$\square: \quad$ Negative semen test for SARS-CoV-2 } \\
\hline : Ong & ing PCR pos & ity in naso & rynx (Con & red as po & e until ne & ve test $r$ & & & & & & & \\
\hline
\end{tabular}

Fig. 1. Timeline of the nasopharyngeal swab and semen sample test for SARS-CoV-2 and test results. The day of admission and hospitalization was accepted as day 1.

men sample was reported as 31 days (IQR 29-36). The authors also investigated the expression profile of ACE2 and transmembrane serine protease 2 (TMPRSS2) within the human testicle and reported that ACE2-mediated viral entry into target host cells within the human testicle is unlikely. The second and similar results were reported by Song et al. [17]. They have shown the absence of SARSCOV-2 in semen samples in 12 recovered patients and in the testes of one deceased patient during acute infection [17]. Similarly, Holtmann et al. [18] reported absence of SARS-CoV-2 in the semen of 34 patients. Only 2 of them were in the acute stage. These results have provided a new and important contribution to the literature about SARS$\mathrm{CoV}-2$. However, the absence of SARS-CoV-2 in semen samples obtained in the post-recovery period is not enough to rule out male genital tract involvement in COVID-19. Investigation of SARS-CoV-2 in semen during the acute stage of the infection is more guiding and decisive for the possibility of male genital system involvement, and for the real risk of sexual transmission. Guo et al. [19] reported that they did not detect SARS-CoV-2 in semen samples in any of 23 adult patients with a recent infection or recovery from COVID-19. Semen samples had been collected after a median 33.5 days (IQR 27.5-33) from confirmation of the diagnosis. It was reported that SARS-CoV-2 was still positive in the sputum and fecal specimens in 12 patients when the semen tested negative. The most potential period for transmission of the virus is the acute stage, where the virus is located at the site of the infection and the viremia may be detected. A recently 
published review has argued that the negative results in studies that failed to detect SARS-CoV-2 in semen may be due to the fact that the samples were not collected at the stage of acute infection [9]. Herein, we demonstrated the absence of SARS-CoV-2 in semen in acute stage of COVID-19. Our study supports the results of previous studies showing the absence of SARS-CoV-2 in semen samples, and it strengthens the thought that the possibility of sexual transmission via semen is unlikely.

Unlike our study and the above-mentioned studies, Li et al. [6] detected SARS-CoV-2 PCR in the semen of 6 of 38 patients (15.8\%). Of 6 patients, 2 were in the recovery stage ( 2 of 23 cases), and 4 were in the acute stage ( 4 of 15 cases). To our knowledge, this is the only study reporting a positive result in semen [6]. However, the method of the study has not been written in detail. The diversity between the results of the studies may be due to differences in disease stage or disease severity, differences in collection and storage standards and viral characteristics. Chen et al. [15] reported that the viremia was related to severe disease. Since the presence of SARS-CoV-2 in semen was generally investigated in mild to moderate cases, there may be a compulsory bias due to the study design. The contamination of semen samples with the patients' respiratory secretions during the sample collection may also be possible and cause a false-positive result if samples are not taken in accordance with sterile conditions [20]. The positivity of semen samples for SARS-CoV-2 should be supported by further studies.

The fact that SARS-CoV-2 was not detected in semen in most of the studies may be related to the low level of viremia. In the previous studies $[1,4,13]$, viremia and viruria were reported to be very low and transient. These results support the argument that SARS-CoV-2 does not aggressively invade non-respiratory sites and is not sexually transmitted. Sexual transmission has been discussed also in other coronaviruses including SARS-CoV. Although it was proposed that sexual transmission is possible since the virus caused testicular damage and germ cell destruction, the transmission by sexual contact remained uncertain in SARS-CoV infection $[21,22]$. Xu et al. [22] reported orchitis as a complication in 6 patients infected with SARS-CoV (G). They did not detect SARS$\mathrm{CoV}$ by in situ hybridizations in the histopathologic examination of the testes but detected extensive IgG precipitation in the seminiferous epithelium. The authors suggested that SARS-CoV may cause orchitis by the autoimmune mechanism in response to viral infection.

The other issue is that genitourinary intervention is risky. The Italian Society of Andrology and Sexual Medi- cine has summarized the available evidence regarding presence of SARS-CoV-2 in semen and stated that the available data do not support the presence of SARSCoV-2 in semen [23]. However, they discussed that this finding may be related to the healing of the infection or that the virus may have never been present in semen and suggested that to confirm or exclude the presence of the virus in semen, studies in currently infected patients are warranted. Our study results show that the presence of the virus is unlikely even in acute infection.

There are some limitations to this study. First, the study was conducted in a relatively limited number of patients and in mild to moderate cases. Secondly, we were unable to investigate the presence of viremia to show the correlation between viremia and seminal fluid results. Thirdly, we could not perform semen parameter analyses in the patients to show the abnormality of semen quality and quantity, other than virus presence.

In conclusion, understanding virus dynamics and knowing all the transmission routes help us to determine preventive measures that have to be taken. Although there is a discrepancy in the literature on this issue, most of the studies showed that SARS-CoV-2 was not detected in semen and sexual transmission via semen is not a possible route. Our study supported the results of the previous studies. The strength of our study is that although semen samples were obtained at the acute stage of infection in all patients when the nasopharyngeal swab test was positive for SARS-CoV-2, the virus could not be detected in semen. We think that our study provides a new finding to fill this gap in the literature.

\section{Acknowledgement}

The authors thanks to Dr. Alperen Korucu for his help.

\section{Statement of Ethics}

The research was conducted ethically in accordance with the World Medical Association Declaration of Helsinki. The study was approved by the Turkish Ministry of Health and Ankara City Hospital, Ethical Committee 1. The ethical approval number is E1/607/2020. A written informed consent was taken from the patients before semen collection.

\section{Conflict of Interest Statement}

The authors have no conflicts of interest to declare. 


\section{Funding Sources}

There are no funding sources to declare.

\section{Author Contributions}

All authors contributed to the review and read and approved the final manuscript. Bircan Kayaaslan wrote the article. Gulay Korukluoglu performed molecular virologic tests.

\section{References}

1 Wu J, Liu J, Li S, Peng Z, Xiao Z, Wang X, et al. Detection and analysis of nucleic acid in various biological samples of COVID-19 patients. Travel Med Infect Dis. 2020 Apr; 101673. Epub ahead of print.

2 Li MY, Li L, Zhang Y, Wang XS. Expression of the SARS-CoV-2 cell receptor gene ACE2 in a wide variety of human tissues. Infect Dis Poverty. 2020 Apr;9(1):45.

3 Chen Y, Guo Y, Pan Y, Zhao ZJ. Structure analysis of the receptor binding of 2019-4 nCoV. Biochem Biophys Res Commun. doi: https://doi.org/10.1016/j.bbrc.2020.02.071.

4 Paoli D, Pallotti F, Colangelo S, Basilico F, Mazzuti L, Turriziani O, et al. Study of 17 SARS-CoV-2 in semen and urine samples of a volunteer with positive naso-18 pharyngeal swab. J Endocrinol Invest. doi: https://doi. org/10.1007/s40618-19 020-01261-1.

5 Pan F, Xiao X, Guo J, Song Y, Li H, Patel DP, et al. No evidence of severe acute respiratory syndrome-coronavirus 2 in semen of males recovering from coronavirus disease 2019. Fertil Steril. 2020 Jun;113(6):1135-9.

6 Li D, Jin M, Bao P, Zhao W, Zhang S. Clinical Characteristics and Results of Semen 21 Tests among Men with Coronavirus Disease 2019. JAMA Netw Open. 2020 May;3(5):e208292. doi: https://doi.org/10.1001/jamanetworkopen.2020.8292.

7 World Health Organization. Coronavirus disease (COVID-19) Dashboard. Available at: https://covid19.who.int/ [accessed 2020 July $15]$.
8 Hui DS, Zumla A. Severe Acute Respiratory Syndrome: Historical, Epidemiologic, and Clinical Features. Infect Dis Clin North Am. 2019 Dec;33(4):869-89.

9 Wang D, Hu B, Hu C, Zhu F, Liu X, Zhang J, et al.Clinical characteristics of 138 hospitalized patients with 2019 novel coronavirus-infected pneumonia in Wuhan, China. JAMA. doi: https://doi.org/10.1001/jama.2020.1585.

10 Goyal P, Choi JJ, Pinheiro LC, Schenck EJ, Chen R, Jabri A, et al. Clinical Characteristics of Covid-19 in New York City. N Engl J Med. 2020 Jun;382(24):2372-2374.

11 Chen Y, Liu Q, Guo D. Emerging coronaviruses: genome structure, replication, and pathogenesis. J Med Virol. 2020 Apr;92(4): $418-23$.

12 Wang W, Xu Y, Gao R, Lu R, Han K, Wu G, et al. Detection of SARS-CoV-2 in Different Types of Clinical Specimens. JAMA. 2020 Mar;323(18):1843-4.

13 Peng L, Liu J, Xu W, Luo Q, Chen D, Lei Z, et al. SARS-CoV-2 can be detected in 13 urine, blood, anal swabs, and oropharyngeal swabs specimens. J Med Virol. doi: https://doi. org/10.1002/jmv.25936.

14 Yu F, Yan L, Wang N, Yang S, Wang L, Tang $\mathrm{Y}$, et al. Quantitative Detection and Viral Load Analysis of SARS-CoV-2 in Infected Patients. Clin Infect Dis. 2020 Jul;71(15):793-8.

15 Chen W, Lan Y, Yuan X, Deng X, Li Y, Cai X, et al. Detectable 2019-nCoV viral RNA in blood is a strong indicator for the further clinical severity. Emerg Microbes Infect. 2020 Feb;9(1):469-73.

16 Lui G, Ling L, Lai CK, Tso EY, Fung KS, Chan $\mathrm{V}$, et al. Viral dynamics of SARS-CoV-2 across a spectrum of disease severity in COVID-19. J Infect. 2020 Aug;81(2):318-56.
17 Song C, Wang Y, Li W, Hu B, Chen G, Xia P, et al. Absence of 2019 novel coronavirus in semen and testes of COVID-19 patients $\dagger$. Biol Reprod. 2020 Jun;103(1):4-6.

18 Holtmann N, Edimiris P, Andree M, Doehmen $\mathrm{C}$, Buest DB, Adams $\mathrm{O}$, et al. Assessment of SARS-CoV-2 in human semen - a cohort study. Fertil Steril. 2020 May;114(2):233-238.

19 Guo L, Zhao S, Li W, Wang Y, Li L, Jiang S, et al. Absence of SARS-CoV-2 in Semen of a COVID-19 Patient Cohort [published online ahead of print, 2020 Jun 29]. Andrology. 2020 Jun;andr.12848.

20 Paoli D, Pallotti F, Turriziani O, Mazzuti L, Antonelli G, Lenzi A, et al. SARS-CoV-2 presence in seminal fluid: myth or reality [published online ahead of print, 2020 May 26]. Andrology. 2020 May;andr.12825.

21 Cardona Maya WD, Du Plessis SS, Velilla PA. SARS-CoV-2 and the testis: similarity with other viruses and routes of infection. Reprod Biomed Online. 2020 Jun;40(6):763-4.

22 Xu J, Qi L, Chi X, Yang J, Wei X, Gong E, et al. Orchitis: a complication of severe acute respiratory syndrome (SARS). Biol Reprod. 2006 Feb;74(2):410-6.

23 Corona G, Baldi E, Isidori AM, Paoli D, Pallotti F, De Santis L, et al. SARS-CoV-2 infection, male fertility and sperm cryopreservation: a position statement of the Italian Society of Andrology and Sexual Medicine (SIAMS) (Società Italiana di Andrologia e Medicina della Sessualità). J Endocrinol Invest. 2020 Aug;43(8):1153-7. 